Document downloaded from:

http://hdl.handle.net/10251/109842

This paper must be cited as:

Hernández-Carrión, M.; Hernando Hernando, MI.; Sotelo-Díaz, I.; Quintanilla-Carvajal, M.; Quiles Chuliá, MD. (2015). Use of image analysis to evaluate the effect of high hydrostatic pressure and pasteurization as preservation treatments on the microstructure of red sweet pepper. Innovative Food Science \& Emerging Technologies. 27:69-78.

doi:10.1016/j.ifset.2014.10.011

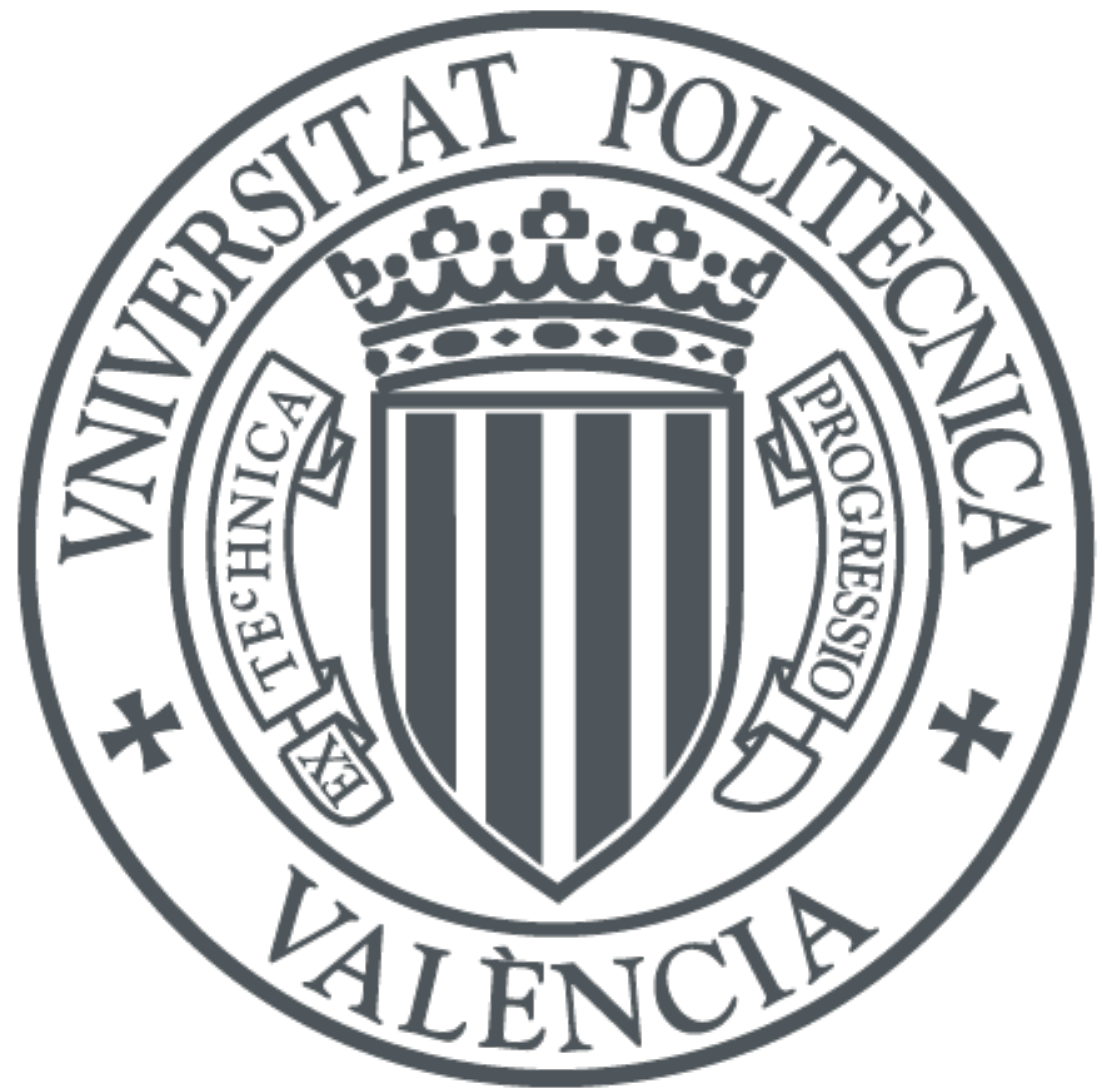

The final publication is available at

https://doi.org/10.1016/j.ifset.2014.10.011

Copyright Elsevier

Additional Information 


\title{
Use of image analysis to evaluate the effect of high hydrostatic pressure and pasteurization as preservation treatments on the microstructure of red sweet pepper
}

\author{
M. Hernández-Carrión ${ }^{\text {a }}$, I. Hernando a ${ }^{\text {a }}$ I. Sotelo-Díaz ${ }^{\text {b }}$, M.X. Quintanilla-Carvajal ${ }^{\text {b,* }}$, A. Quiles ${ }^{\text {a }}$ \\ a Research Group of Food Microstructure and Chemistry, Department of Food Technology, Universitat Politècnica de València, Camino de Vera s/n, Valencia 46022, Spain \\ b Ingeniería de Producción Agroindustrial, Facultad de Ingeniería, Universidad de La Sabana, Campus Puente del Común, Autopista Norte de Bogotá, Chía, Cundinamarca, Colombia
}

\section{A R T I C L E I N F O}

\section{Keywords:}

High hydrostatic pressure

Image analysis

Microstructure

Pasteurization

Red pepper

\begin{abstract}
A B S T R A C T
The aim of this work was to evaluate the effect of HHP treatment and PAST on the microstructure of red Lamuyotype sweet peppers using image analysis and to determine the parameters that allow characterizing the changes observed on the structure using different magnifications $(100 \times, 200 \times$, and $350 \times)$. The results show that all the preservation treatments evaluated caused structural modifications on the microstructure of red sweet pepper, but HHP at $500 \mathrm{MPa}$ and PAST had less impact. Fractal dimension texture, contrast, inverse difference moment, and entropy are texture features that are appropriate for characterizing the effect of HHP and PAST on red pepper texture. In this context, it is important to consider the magnification at which red pepper texture is evaluated because cell damage caused by treatments is best observed at low magnification. Consequently, image analysis could be used in future studies to relate microstructure to the functionality of products subject to HHP.

Industrial relevance: Red sweet peppers (Capsicum annuum) are an excellent source of essential nutrients and bioactive compounds. High hydrostatic pressure (HHP) applied during food processing can improve the retention of food quality attributes and nutritional and organoleptic properties better than pasteurization (PAST). Image analysis is a non-invasive technique that allows to provide objective evaluations from digitalized images. There are no studies that quantify the effect of HHP and PAST using image texture parameters and that evaluate the effect of the magnification used on these texture features. These features are critical factors that determine food acceptance or rejection by the consumers. Thus, texture measurement has gained much attention from food science and industry. Therefore, it would be interesting to study the effect of these treatments on the microstructure of red sweet pepper tissue using image analysis. Thereby, it would be possible to relate the image information to structural modifications and to the extractability of bioactive compounds or acceptance of preservation processes by consumers.
\end{abstract}

\section{Introduction}

Red sweet peppers (Capsicum annuum $\mathrm{L}$ ) are an excellent source of essential nutrients such as carbohydrates, vitamins, and minerals (Faustino, Barroca, \& Guiné, 2007). In addition to being a good source of essential nutrient, pepper is rich in fiber and other bioactive compounds such as carotenoids, which possess antioxidant and antiinflammatory activity, phenols, and flavonoids (Deepa, Kaur, George, Singh, \& Kapoor, 2007; Duma \& Alsina, 2012; Zhuang, Chen, Sun, \& Cao, 2012). Moreover, fresh pepper is considered to be one of the vegetables with the highest content of vitamin $C$ within the plant kingdom (Serrano et al., 2010). Additionally, pepper prevents atherosclerosis and hemorrhages, improves scar formation and stamina, and improves blood cholesterol levels (Faustino et al., 2007).
The demand for safe foods that possess sensory freshness characteristics and biological properties that go beyond the strictly nutritional have led researchers and manufacturers to develop new processing and conservation technologies. Of these new technologies, high hydrostatic pressure (HHP) is one of the most economically viable of what are known as non-thermal treatments (Devlieghere, Vermeiren, \& Debevere, 2004; Rastogi, Raghavarao, Balasubramaniam, Niranjan, \& Knorr, 2007). HHP facilitates the production of food products that have the quality of fresh foods but the convenience and profitability associated with shelf life extension (McClements, Patterson, \& Linton, 2001). HPP can be applied to a range of different foods, including juices and beverages, fruits and vegetables, meat-based products, fish, and precooked dishes, with meat and vegetables being the most popular applications (Norton \& Sun, 2008).

On the other hand, image analysis can be particularly a useful tool for characterizing food morphology because the highly irregular structures of many food materials elude precise quantification by conventional means. This technique allows to obtain measurements from digitalized images. These measurements provide objective evaluations of the 
morpho-colorimetric features of samples, a method that is more quantitative and less biased than the common method of visual perception, which is prone to variation due to the personal opinions of inspectors (Russ, 2007; Sonka, Hlavac, \& Boyle, 2008; Sun, 2008). Nowadays, many software programs for image processing and analysis are available on the market able to analyze digital images in real time providing precise and accurate measurements of the size, shape, color, and texture of the objects studied. Many studies have demonstrated the utility of image analysis for the study of morphometric and colorimetric characteristics in fruits and vegetables (López-García, Andreu-García, Blasco, Aleixos, \& Valiente, 2010; Rocha, Hauagge, Wainer, \& Goldenstein, 2010), in legumes and durum wheat kernels (Venora, Grillo, Ravalli, \& Cremonini, 2009; Venora, Grillo, \& Saccone, 2009; Venora, Grillo, Shahin, \& Symons, 2007), in and bakery products (Abdullah, Aziz, \& Dos Mohamed, 2000; Grillo et al., 2014).

Image analysis can evaluate different morphometric characteristics, also known as morphometric features. Morphometric features can be divided into three groups: dimensionality, which refers to measuring various characteristics of the object such as area, perimeter, Feret diameter, and maximum length, among others, using specific computer programs; form, which refers to the graphical representation of the study object as an approximate reference to an Euclidean geometric figure, including circular and elliptical form factors, eccentricity, sphericity, and convexity; and texture, which refers to the information needed to describe the regularity of an object, e.g., its compactness, roughness and sinuosity (Aguilera, 2007).

Texture is one of the important characteristics used in identifying objects or regions of interest in an image, whether the image be a photomicrograph, an aerial photograph, or a satellite image (Haralick, Shanmugam, \& Dinstein, 1973). The gray level co-occurrence matrix (GLCM) proposed by Haralick et al. (1973) is an image processing technique that has been widely used for measuring of texture in images. It first generates a gray level co-occurrence matrix that is defined as the distribution of co-occurring values at a given offset over a given image then calculate a set of textual features (usually called Haralick features) from the matrix that can reflect the image texture. Different textural features can be obtained from an image using image analysis, such as angular second moment, contrast, correlation, inverse difference moment, and entropy.

Although numerous publications can be found about the effect of different preservation treatments, such as high hydrostatic pressure and pasteurization (PAST) on the microstructure and on the size and/ or shape parameters of different plant tissues (Hernández-Carrión, Hernando, \& Quiles, 2014; Hernández-Carrión, Vázquez-Gutiérrez, Hernando, \& Quiles, 2014; Vázquez-Gutiérrez, Hernández-Carrión, Quiles, Hernando, \& Pérez-Munuera, 2012; Vázquez-Gutiérrez et al., 2013) and about the effect of different processing techniques such as drying on the color of sweet pepper using image analysis (Romano, Argyropoulos, Nagle, Khan, \& Müller, 2012), there are no studies that quantify the effect of HHP and PAST using image texture parameters and that evaluate the effect of the magnification used on these texture parameters. In this sense, the study of the effect of HHP and PAST on the microstructure of red sweet pepper tissue using image analysis is essential and would enable to relate the image information to structural modifications and to the extractability of some bioactive compounds, such as carotenoids, that could affect the functionality of the selected plant tissue. Also, it is important to start to develop appropriate decision algorithms, methods, and magnifications that allow acceleration and optimize industrial processes that can be evaluate by image analysis, particularly in the case of important products as sweet pepper.

The aim of this work was (1) to evaluate the effect of HHP treatment and PAST on the microstructure of red Lamuyo-type sweet peppers using image analysis and (2) to determine the parameters that allow characterizing or quantitatively describe the changes observed on the structure using different magnifications.

\section{Materials and methods}

\subsection{Plant material and sample preparation}

The plant material used was red Lamuyo-type sweet peppers at commercial maturity stage. The red peppers, acquired from a local market in September 2013, were washed, cut into pieces measuring about $15 \mathrm{~mm}$ along each side and heat-sealed in $200 \times 200 \mathrm{~mm}$ plastic bags (Doypack type, Amcor, Spain). Each bag contained approximately $100 \mathrm{~g}$ of red sweet pepper. One batch was not subjected to any treatment (CNT). The second, third, fourth, and fifth batch were treated by HHP at different pressures $(100,200,300$, and $500 \mathrm{MPa})$ according to Hernández-Carrión et al. (2014). The last batch was pasteurized (PAST) in a water bath at $70{ }^{\circ} \mathrm{C}$ for $10 \mathrm{~min}$ (come-up time to temperature $=30 \mathrm{~min}$ ) (Hernández-Carrión et al., 2014). The bags were then stored at $4{ }^{\circ} \mathrm{C}$ until they were analyzed. The microstructure was analyzed within $24 \mathrm{~h}$ of treatment.

\subsection{High hydrostatic pressure (HHP) treatments}

Bags with approximately $100 \mathrm{~g}$ of red sweet pepper were placed inside a hydrostatic pressure unit with a $135 \mathrm{~L}$ capacity (Hyperbaric type 135, Burgos, Spain), using water as the pressure medium. Different HHP treatments were studied, coded T1 (100 MPa), T2 (200 MPa), T3 (300 MPa), and T4 (500 MPa) during $15 \mathrm{~min}$ at $25^{\circ} \mathrm{C}$.

\subsection{Microstructure analysis}

\subsubsection{Light microscopy (LM)}

For the LM, the samples $\left(2 \mathrm{~mm}^{3}\right)$ were fixed with a $25 \mathrm{~g} \mathrm{~L}^{-1}$ glutaraldehyde solution $\left(0.025 \mathrm{M}\right.$ phosphate buffer, $\left.\mathrm{pH} 6.8,4^{\circ} \mathrm{C}, 24 \mathrm{~h}\right)$, postfixed with a $20 \mathrm{~g} \mathrm{~L}^{-1} \mathrm{OsO}_{4}$ solution $(1.5 \mathrm{~h})$, dehydrated using a graded ethanol series $\left(300,500\right.$ and $\left.700 \mathrm{~g} \mathrm{~kg}^{-1}\right)$, contrasted in $20 \mathrm{~g} \mathrm{~L}^{-1}$ uranyl acetate, dehydrated with ethanol ( 960 and $1000 \mathrm{~g} \mathrm{~kg}^{-1}$ ), and embedded in epoxy resin (Durcupan; Sigma-Aldrich, St. Louis, MO, USA) at $65.5^{\circ} \mathrm{C}$ for $72 \mathrm{~h}$. The samples were cut using a Reichert Jung ultramicrotome (Leica Mycrosystems, Wetzlar, Germany). Semi-thin sections $(1.5 \mu \mathrm{m})$ were stained with toluidine blue and examined under a Nikon Eclipse 80i light microscope (Nikon, Tokyo, Japan).

\subsubsection{Scanning electron microscopy (SEM)}

Pieces ( $3 \mathrm{~mm}$ wide) from raw and treated red sweet pepper were frozen at $-20^{\circ} \mathrm{C}$ and then freeze-dried at 1 Pa for 3 days (LIOALFA- 6, Telstar, Barcelona, Spain). Then red sweet pepper samples were vacuum sealed in vials in the same freeze-drier so that they would remain stable (Llorca et al., 2001). After that, they were individually placed on SEM slides with the aid of colloidal silver and then gold-coated with (SCD005, Baltec, Germany) at $10^{-2} \mathrm{~Pa}$ and an ionization current of $40 \mathrm{~mA}$ for $120 \mathrm{~s}$. The samples were observed in a scanning electron microscope (JSM-5410, Jeol, Japan) at an acceleration voltage of $15 \mathrm{kV}$. The microscope was equipped with an integrated program for digital image capture (INCA 4.09, Oxford Instruments, England). Magnifications of $100 \times, 200 \times$, and $350 \times$ were used.

\subsection{Image analysis}

\subsubsection{Morphometric analysis}

Image processing was carried out according to Pedreschi, Mery, Mendoza, and Aguilera (2004). Images of the red sweet pepper of $1280 \times 1024$ pixels were captured using a light microscopy and stored as bit maps (gray scale with brightness values between 0 and 255) (Quintanilla-Carvajal et al., 2011) by using the ImageJ software (Rasband, W.S., ImageJ v.1.43 s, National Institute of Health, Bethesda, Maryland, USA). The following morphological parameters were determined for each treatment: area, perimeter, circularity, and Feret diameter. With these results, the cell size distribution was evaluated. 


\subsubsection{Texture image analysis}

Images of the red sweet pepper of $1024 \times 786$ pixels were captured using an electronic microscopy and stored as bitmaps in a gray scale with brightness values between 0 and 255 for each pixel constituting the image. A generalization of the box counting method was used to evaluate the fractal dimension of the images (FDt). In this work, the shifting differential box-counting method (SDBC) (Chen, Yuan, Hsiao, \& Hsieh, 2001) was used to evaluate the fractal dimension of texture of SEM images using the Image 1.34 software. Three different crops at the three different magnifications used $(100 \times, 200 \times$, and $350 \times)$ were evaluated for each treatment. The size of the crops was the same for all the evaluated magnifications $(270 \mu \mathrm{m} \times 270 \mu \mathrm{m})$.The texture parameters (angular second moment, contrast, correlation, inverse difference moment, and entropy) of SEM images were evaluated using the GLCM and surface plot tools of ImageJ.

The textural feature angular second moment, also called energy, measures the texture uniformity or orderliness of an image (Ou, Pan, \& Xiao, 2014). Higher angular second moment values indicate more directional uniformity in the image (Yang, Beyenal, Harkin, \& Lewandowski, 2000). The angular second moment for grayscale image is defined as (Agüera, Aguilar, \& Aguilar, 2008):

Angular second moment $=\operatorname{sum}(\mathrm{p}(\mathrm{i}, \mathrm{j}))^{2}$

The textural feature contrast is a measure of the intensity contrast between a pixel and its neighbor over the whole image. It measures the local variation in the GLCM. Contrast can be seen as dynamic range of gray level or sharpness of edges. The range of contrast lies between 0 and (size (GLCM, 1), -1$)^{2}$. Furthermore, contrast is 0 for a constant image (Laddi, Sharma, Kumar, \& Kapur, 2013).

The textural feature correlation is a measure of how correlated a pixel is to its neighbor over the whole image. Its range lies between -1 and +1 . Also, the correlation is 1 or -1 for a perfectly positively or negatively correlated image. Correlation measures the joint probability of occurrence of pixel pairs of GLCM (Laddi et al., 2013).

The textural feature inverse difference moment (IDF) is a similar measure to the angular second moment but normalized for distance. Higher inverse difference moment values indicate more or less variation in image contrast (Yang et al., 2000).

The textural feature entropy was determined by color to grayscale conversion of acquired tea sample images, whereas other features were calculated by conversion of grayscale images into GLCM, which is created by calculating how often a pixel with gray-level (grayscale intensity) value $i$ occurs horizontally adjacent to a pixel with the value $j$. Each element $(i, j)$ in GLCM specifies the number of times that the pixel with value i occurred horizontally adjacent to a pixel with value $j$ (Haralick \& Shapiro, 1992; Haralick et al., 1973). Entropy is a statistical measure of randomness that can be used to characterize the texture of the input image. Entropy for grayscale image is defined as

Entropy $=-\operatorname{sum}(p(i, j)) \cdot * \log _{2}(p(i, j))$

where $p$ contains the histogram counts used for 256 bins of grayscale image (Gonzalez, Woods, \& Eddins, 2003). Entropy is highest when all entries in $p(i, j)$ are of similar magnitude and small when the entries in $p(i, j)$ are unequal (Laddi et al., 2013).

\subsection{Experimental design and statistical analysis}

\subsubsection{Morphometric parameters}

An analysis of variance (one-way ANOVA) was applied to study the differences between the treatments (CNT, T1, T4, and PAST) for the morphometric data; the least significant differences were calculated by Fisher's test, and the significance at $p<0.05$ was determined.

\subsubsection{Texture parameters}

A categorical multifactorial experimental design with two factors: treatment (CNT, T1, T2, T3, T4, and PAST) and magnification $(100 \times$, $200 \times$, and $350 \times$ ) was used. Analysis of variance (ANOVA) was performed on the texture data using the Statgraphics Plus version 5.1 software package (Statistical Graphics Co., Rockville, MD, USA). The least significant differences (LSD) were calculated by Fisher's test, and the significance at $p<0.05$ was determined.

\section{Results and discussion}

\subsection{Light microscopy (LM)}

The parenchyma of the untreated red Lamuyo-type sweet peppers was composed of turgid cells round or semi-round in appearance with well-stained and well-defined cell walls (Fig. 1A). The intercellular spaces were mostly triangular and packed with solutes. High carotenoid pigment content, accumulated inside the chromoplasts, could also be seen in the interior of the cells.

When the red pepper was subjected to HHP treatment at pressures of $100 \mathrm{MPa}$ (treatment T1, Fig. 1B), its tissues broke down completely. The cell walls were lightly blue-stained. Areas where the cell walls had degraded completely could be seen throughout the parenchymal tissue. However, these areas were occupied by clumps of matter organized like bridges connecting the other walls to each other, maintaining the continuity and the boundaries of the cells.

The HHP at 500 MPA (treatment T4, Fig. 1C) led to visible structural changes in the parenchyma compared with the untreated pepper (Fig. 1A) but less tissue breakdown than with the HHP T1 treatment (Fig. 1B). The red sweet pepper parenchymal cells subjected to HHP T4 were found to be round in shape and their cell walls were well stained.

The thermal treatment (PAST, Fig. 1D), although led to structural modifications, caused less breakdown of the parenchymal tissue than the HHP treatments. The cells were more lightly stained than in the untreated pepper (Fig. 1A). Separation between the cell walls of adjoining cells only took place in some areas giving place to irregular intercellular spaces.

Hence, it can be concluded that each preservation treatment (HHP and PAST) caused different tissue architecture, which suggest that the mechanisms of cell permeabilization are not the same (Tedjo, Taiwo, Eshtiaghi, \& Knorr, 2002; Vega-Gálvez et al., 2011).

\subsubsection{Morphometric analysis}

Table 1 shows the results obtained for the evaluated morphometric parameters (area, perimeter, circularity, and Feret diameter). Statistical analysis of the results revealed that the CNT red pepper had significantly lower circularity values $(p<0.05)$, while PAST peppers had significantly higher area, perimeter, and Feret diameter $(p<0.05)$. By contrast, there were no statistically significant differences $(p>0.05)$ between the CNT, $\mathrm{T} 1$, and $\mathrm{T} 4$ red peppers with respect to area, perimeter, or Feret diameter. There were no statistically significant differences $(p>0.05)$ in circularity between cells of T1, T4, and PAST peppers. Thus, it seems that HHP and PAST promote an increase in the circularity of pepper cells. Trejo Araya et al. (2007) obtained different results when studying the effect of HHP on carrot microstructure. Those authors found that HHP decreased the circularity of cells compared with fresh carrot cells. By contrast, Penna, Subbarao, and Barbosa-Cánovas (2007) studied the effect of high-pressure treatment on the micelle microstructure of yogurt. They found that micelles in high-pressure milks were more spherical in shape and present more uniform size distribution compared to heat-treated milks.

However, only PAST caused statistically significant changes $(p<$ 0.05 ) in area, perimeter, and Feret diameter in red pepper cells, contradicting the observations in the micrographs (Fig. 1) where treatments $\mathrm{T} 4$ and (to a greater degree) T1 appeared to cause greater changes in 


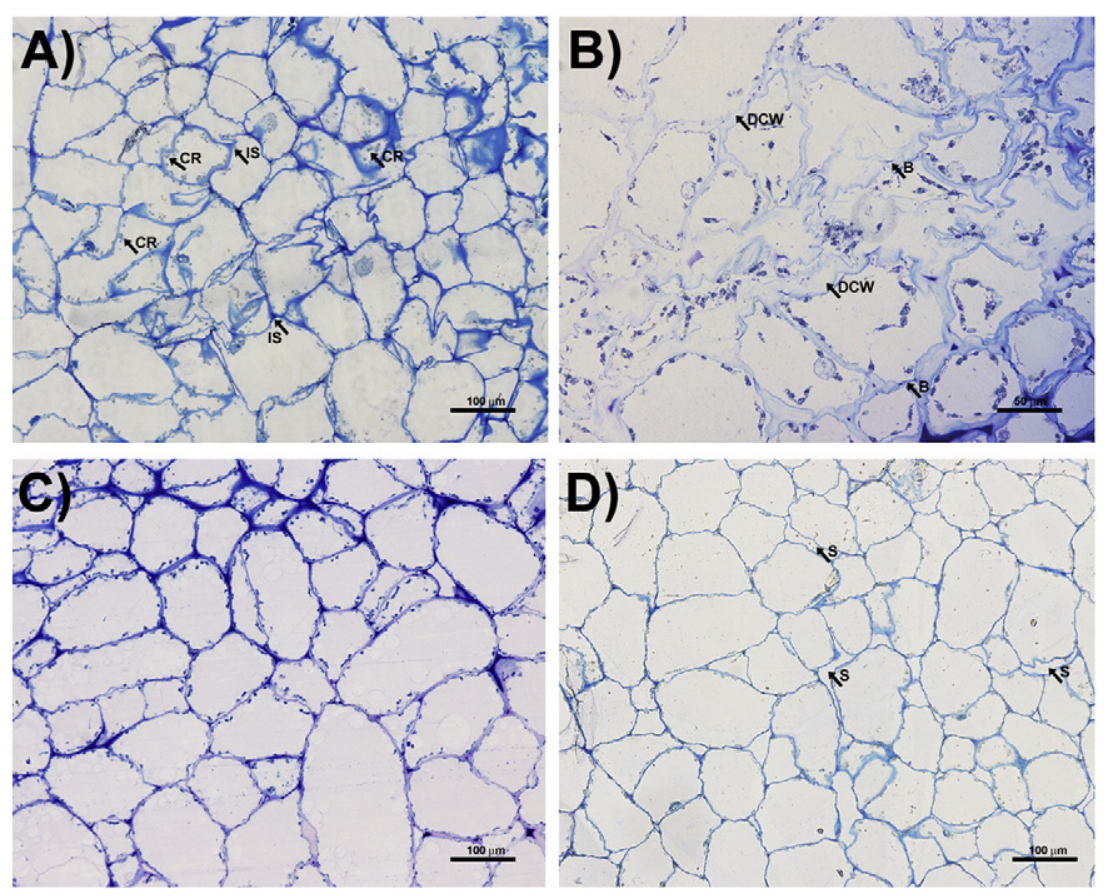

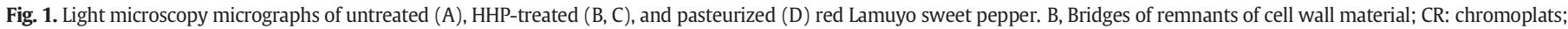
DCW: degraded cell wall; IS, intercellular space; S, separation between cell walls. Magnification: $20 \times(A), 10 \times(B, C$, and D).

the red pepper tissue microstructure than PAST. Therefore, it can be concluded that HHP does not result in significant changes in the evaluated morphometric parameters despite noteworthy microstructural changes in the micrographs of peppers subjected to HHP (including less staining and more degraded cell walls). This phenomenon could be explained by the fact that less aggressive treatments from a microstructural point of view (T4 and PAST) could allow water to migrate radially and in a controlled fashion, thus favoring the maintenance of cell shape. It is also interesting to note that Hernández-Carrión et al. (2014) found that these treatments (T4 and PAST) least affected carotenoids, antioxidant capacity, dietary fiber, and red pepper texture while promoting preservation and functionality of the plant material when studying the effect of different HHP and PAST treatments on bioactive compounds and red pepper texture. These results suggest that the lower the structural damage caused by a treatment, the less impact on the functionality of the pepper. Similarly, Hernández-Carrión et al. (2014) found that PAST caused an increase in the area of red pepper cells when studying the microstructure of Lamuyo red peppers subjected to different preservation treatments (HHP and PAST), obtaining values of $12,127 \mu \mathrm{m}^{2}$ compared with CNT red pepper $\left(9,662 \mu \mathrm{m}^{2}\right)$.

Fig. 2 shows the effects of applying HHP at $100 \mathrm{MPa}$ (T1) and $500 \mathrm{MPa}$ (T4) as well as the effect of PAST on the cell size distribution.
The figure indicates that CNT peppers show two populations of easily distinguished cell sizes, one with cells having a $125 \mu \mathrm{m}$ Feret diameter and the other with cells having a $160 \mu \mathrm{m}$ Feret diameter. When the pepper was subject to treatment $\mathrm{T} 1$, the proportion of cells with the greater diameter tended to decrease with respect to the CNT pepper, which caused widening of the curve, thus encompassing a greater range of cell diameters. Similarly, when applying treatment T4, the curve of the distribution of the cell diameters appeared to widen relative to the CNT pepper, covering a greater range of diameters and coinciding with most of the cell population of the first peak of the CNT pepper (approx. $125 \mu \mathrm{m}$ ). As shown in Fig. 2, by contrast, when peppers are subjected to PAST, their cell diameter increases; in this figure, the curve corresponding to PAST is slightly shifted to the right, and its maximum value coincides with the second peak of the CNT pepper (approx. $160 \mu \mathrm{m}$ ). In this case, and as a result of the increased cell size caused by the PAST treatment, the proportion of cells with the larger diameter decreases relative to the CNT and T4 treatment peppers. This result coincides with the result obtained by analyzing the morphometric parameters of peppers subjected to different treatments (Table 1) in which it was observed that the PAST peppers had a significantly higher Feret diameter $(p<0.05)$. It appears that the distribution of the cell diameters of red peppers varies depending on the HHP or PAST treatment. Whereas

Table 1

Morphometric parameters of the cells of untreated, HHP-treated, and pasteurized red Lamuyo sweet pepper.

\begin{tabular}{|c|c|c|c|c|}
\hline & Area $\left(\mu \mathrm{m}^{2}\right)$ & Perimeter $(\mu \mathrm{m})$ & Circularity & Feret diameter $(\mu \mathrm{m})$ \\
\hline $\mathrm{CNT}$ & $\begin{array}{l}9325.2^{\mathrm{a}} \\
(2790.6)\end{array}$ & $\begin{array}{l}408.1^{\mathrm{a}} \\
(70.9)\end{array}$ & $\begin{array}{l}0.7^{\mathrm{a}} \\
(0.1)\end{array}$ & $\begin{array}{l}140.3^{\mathrm{a}} \\
(20.8)\end{array}$ \\
\hline $\mathrm{T} 1$ & $\begin{array}{l}10186.9^{a} \\
(5577.2)\end{array}$ & $\begin{array}{l}399.8^{\mathrm{a}} \\
(111.1)\end{array}$ & $\begin{array}{l}0.8^{\mathrm{b}} \\
(0.1)\end{array}$ & $\begin{array}{l}143.5^{\mathrm{a}} \\
(42.3)\end{array}$ \\
\hline $\mathrm{T} 4$ & $\begin{array}{l}10524.5^{\mathrm{a}} \\
(6038.7)\end{array}$ & $\begin{array}{l}400.8^{a} \\
(119.2)\end{array}$ & $\begin{array}{l}0.8^{\mathrm{b}} \\
(0.1)\end{array}$ & $\begin{array}{l}143.6^{\mathrm{a}} \\
(38.0)\end{array}$ \\
\hline PAST & $\begin{array}{l}13722.6^{b} \\
(2690.4)\end{array}$ & $\begin{array}{l}469.3^{b} \\
(55.6)\end{array}$ & $\begin{array}{l}0.8^{\mathrm{b}} \\
(0.1)\end{array}$ & $\begin{array}{l}164.6^{\mathrm{b}} \\
(20.1)\end{array}$ \\
\hline
\end{tabular}

The values in parenthesis are the standard deviations.

In the same column, means without the same letter reveal significant differences $(p<0.05)$ according to the LSD multiple range test. 


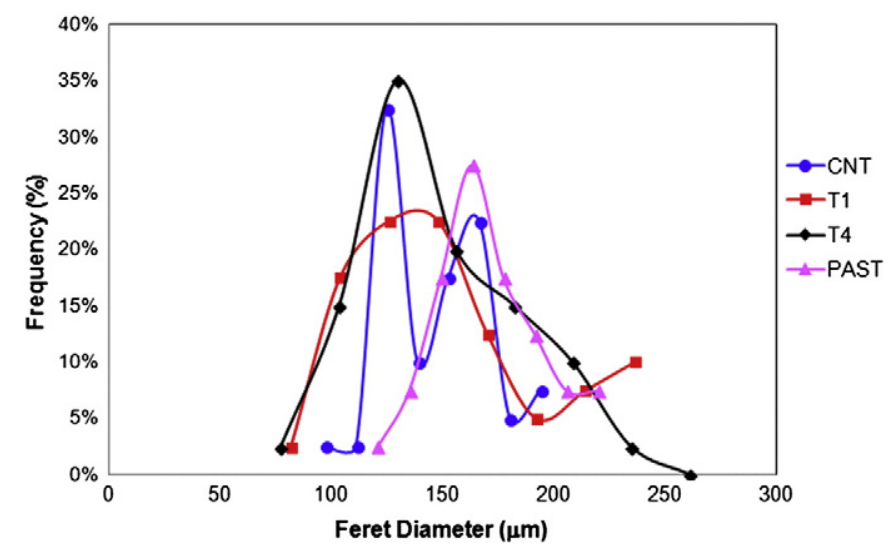

Fig. 2. Particle size distribution of the cells of untreated, HHP-treated, and pasteurized red Lamuyo sweet pepper.

HHP tends to widen the curve, PAST tends to shift it to the right. The widening observed upon applying the T1 and T4 treatments could be explained by microstructural changes caused by their application (Fig. 1B and D), which would trigger disruption of the cells and thus promote union between them, increasing their size. It is worth noting that the increase in cell diameter observed in Fig. 2 was not statistically significant $(p>0.05)$ when taking into account the results shown in Table 1.

\subsection{Scanning electron microscopy (SEM)}

The parenchymal tissue of the CNT pepper (Fig. 3A-C) was characterized by turgid cells of rounded and semi-rounded appearance intimately linked to one another with little intercellular space. Both treatments (HHP and PAST) caused visible microstructural changes. However, HHP at low and medium pressures, i.e., the T1 (Fig. 3D-F), T2 (Fig. 3G-I), and T3 (Fig. 3J-L) treatments, led to more dramatic changes in the red pepper tissue. In these cases, a completely waterlogged tissue was observed in which different cells could be hardly distinguished, although they were clearly differentiated in the CNT pepper. HHP T4 treatment (Fig. 3M-O) and PAST (Fig. 3P-R) caused less dramatic microstructural changes in the red peppers. Thus, in these cases, it was possible to distinguish rounded and semi-rounded cells that were slightly separated from one another, resulting in intercellular spaces filled with air. These results are consistent with the results observed when studying the microstructure of samples through light microscopy where it was observed that $\mathrm{T} 1$ treatment at the lowest pressure caused the greatest structural changes. While the HHP T4 and PAST treatments did cause microstructural changes, they had less impact on the tissue structure of the red peppers.

Fig. 4 shows cropped images $(270 \mu \mathrm{m} \times 270 \mu \mathrm{m})$ that were considered for determining the image texture parameters for each of the studied treatments (CNT, T1, T2, T3, T4, and PAST) at the three magnifications used $(100 \times, 200 \times$, and $350 \times)$ during micrograph acquisition. It is important to note that the treatments that caused greater cellular changes (T1, T2, and T3) showed a rough and irregular surface at the different magnifications studied, while the surface of the CNT pepper and the other treatments that best preserved red pepper structure (T4 and PAST) were characterized as homogenous in which holes could be distinguished that corresponded to areas occupied by the cells that make up the red pepper tissue. These results agree with the results obtained from the microstructural study (Fig. 3) in which it was observed that treatments $\mathrm{T} 1, \mathrm{~T} 2$, and $\mathrm{T} 3$ had a greater impact on the red pepper structure. Similarly, the T4 and PAST treatments preserved the structure best; these peppers were most similar to the CNT pepper in that some of the cells could be clearly distinguished.

\subsubsection{Texture image analysis}

The statistical analysis of the results revealed a significant interaction $(p<0.05)$ between the applied treatment and the magnification used when analyzing the texture fractal dimension (FDt) of the Lamuyo red pepper micrographs. In this sense, it can be observed (Fig. 5A) that there were no statistically significant differences $(p>0.05)$ between the micrographs of the CNT, PAST, and HHP T4 peppers when using $100 \times$ magnification. However, statistically significant differences $(p<0.05)$ were found when pressures of 100 (T1), 200 (T2), and 300 (T3) MPa were applied, and an increase in pressure tended to significantly decrease the FDt of the Lamuyo red pepper micrographs $(p<0.05)$. This result was related to the decreased structural damage observed with increasing pressure. The treatments with higher FDt values (T1, T2, and T3) were also shown to have a rougher and more irregular surface, suggesting that rougher surfaces have higher FDt values (Arzate-Vázquez et al., 2012; Quevedo, Jaramillo, Díaz, Pedreschi, \& Aguilera, 2009). Similar results were obtained by Quevedo, López, Aguilera, and Cadoche (2002) when studying the FDt and the surface of chocolate and pumpkin. The authors found that pumpkin had higher FDt values and a rougher surface compared with chocolate, which had lower FDt values and a more homogenous surface. In the same way, Gonzales-Barron and Butler (2008) related the higher FDt values to more roughness surfaces of bread crumbs images and Barrera et al. (2013) obtained in starch granules that damaged ones showed higher FDt values than native ones. When the $200 \times$ magnification was used, no statistically significant difference $(p>0.05)$ in FDt was found between the micrographs of the CNT and the T4 peppers, while the other treatments showed significantly higher $(p<0.05)$ FDt values. However, the increased pressure did not show the same effect as when $100 \times$ magnification was used. T2 treatment resulted in significantly higher $(p<0.05)$ FDt values than T1 treatment, which was in contrast to the results when using $100 \times$ magnification. Finally, when using $350 \times$ magnification, no statistically significant difference $(p>0.05)$ in the FDt values was found between the micrographs of the CNT, PAST, and T2 and $\mathrm{T} 4$ treatment peppers. Treatments $\mathrm{T} 1$ and $\mathrm{T} 3$ had significantly higher $(p<0.05)$ FDt values, which shows that the more homogenous surfaces (CNT, T4, and PAST) tended to have lower FDt values. It thus appears that the magnification used to study the microstructure of the red pepper has a clear influence on the texture parameters determined from the micrographs. However, increased magnification did not exert the same effect on all of the treatments. Thus, for the CNT, PAST, T3, and T4 peppers, the increase in magnification resulted in a significant increase $(p<0.05)$ in the FDt values of the micrographs. The structural damage of sweet pepper caused by treatment is best observed at smaller scales, so in this case, the best magnification to select would be $100 \times$. Anyway, it would be recommendable to investigate other magnifications in future research. Similar results were obtained by Quintanilla-Carvajal et al. (2011) when studying the FDt in $\alpha$-tocopherol agglomerated microcapsules at two different magnifications $(500 \times$ and $2000 \times)$, where the FDt values were higher at $2000 \times$ than at $500 \times$. The opposite behavior was observed when treatments T1 and T2 were applied: significantly lower $(p<0.05)$ FDt values were obtained at $350 \times$ magnification compared with $100 \times$ and $200 \times$. Hence, FDt seems to be a useful textural parameter to numerically describe microstructural changes. Numerous publications can be found in the literature that indicate this fact (Barrera et al., 2013; Ersahin, Gunal, Kutlu, Yetgin, \& Coban, 2006; Gonzales-Barron \& Butler, 2008; Quevedo et al., 2002, 2009; Quintanilla-Carvajal et al., 2011; Tang \& Marangoni, 2006; Valous, Mendoza, Sun, \& Allen, 2009).

When analyzing the angular second moment (Fig. 5B) of the micrographs from red peppers subjected to different preservation treatments, no statistically significant interaction $(p>0.05)$ was found between the studied parameters, and there was no statistically significant difference $(p>0.05)$ in the textural properties for any of the evaluated parameters (treatment and magnification). It thus appears that this parameter is not optimal for evaluating the effect of HHP and PAST on the image texture of red peppers. Several studies have shown the pertinence of this textural parameter to study the texture of different type of surfaces as 

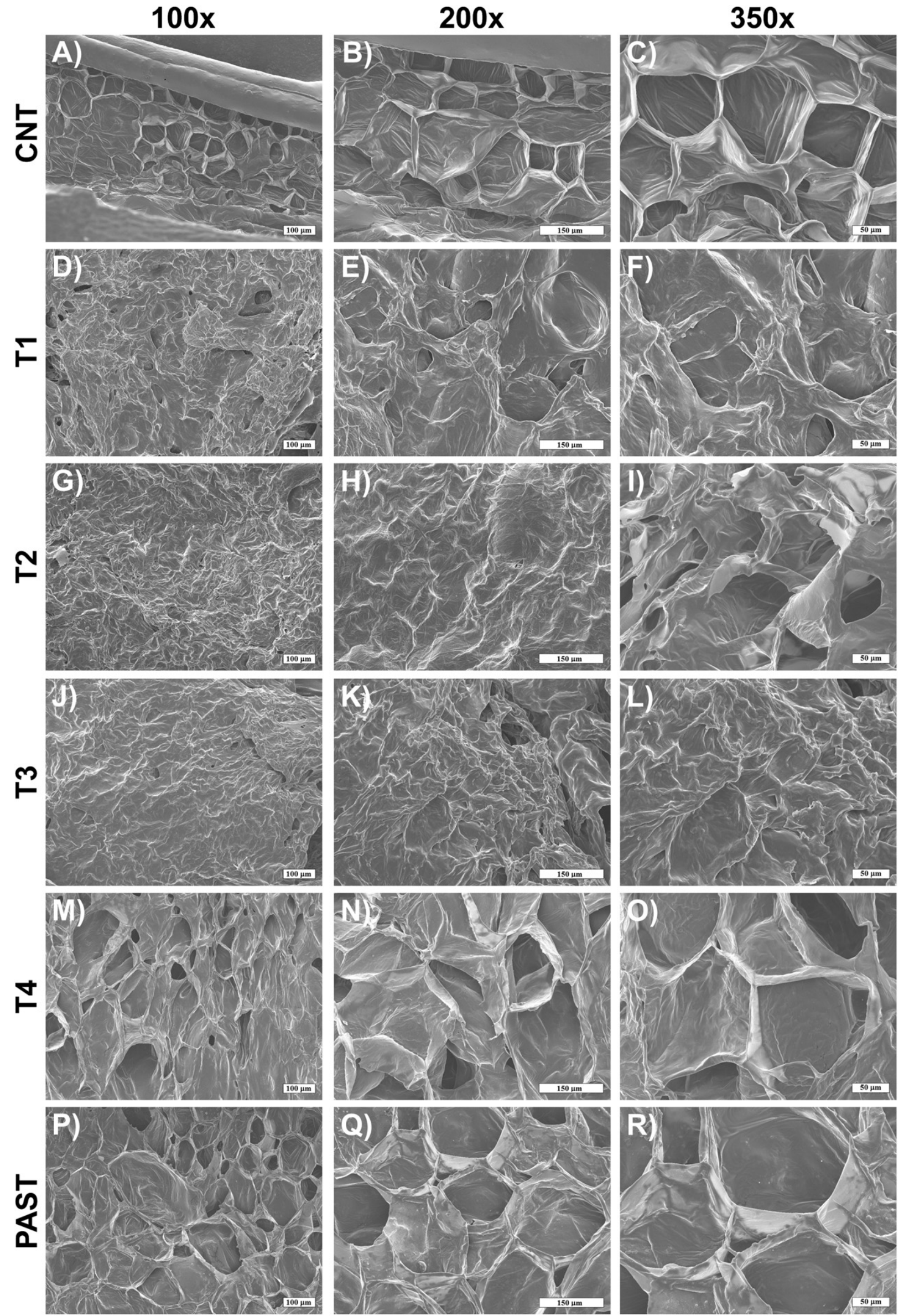

Fig. 3. Scanning electron microscopy micrographs of untreated, HHP-treated, and pasteurized red Lamuyo sweet pepper. Magnification: 100×, 200×, and 350×. 


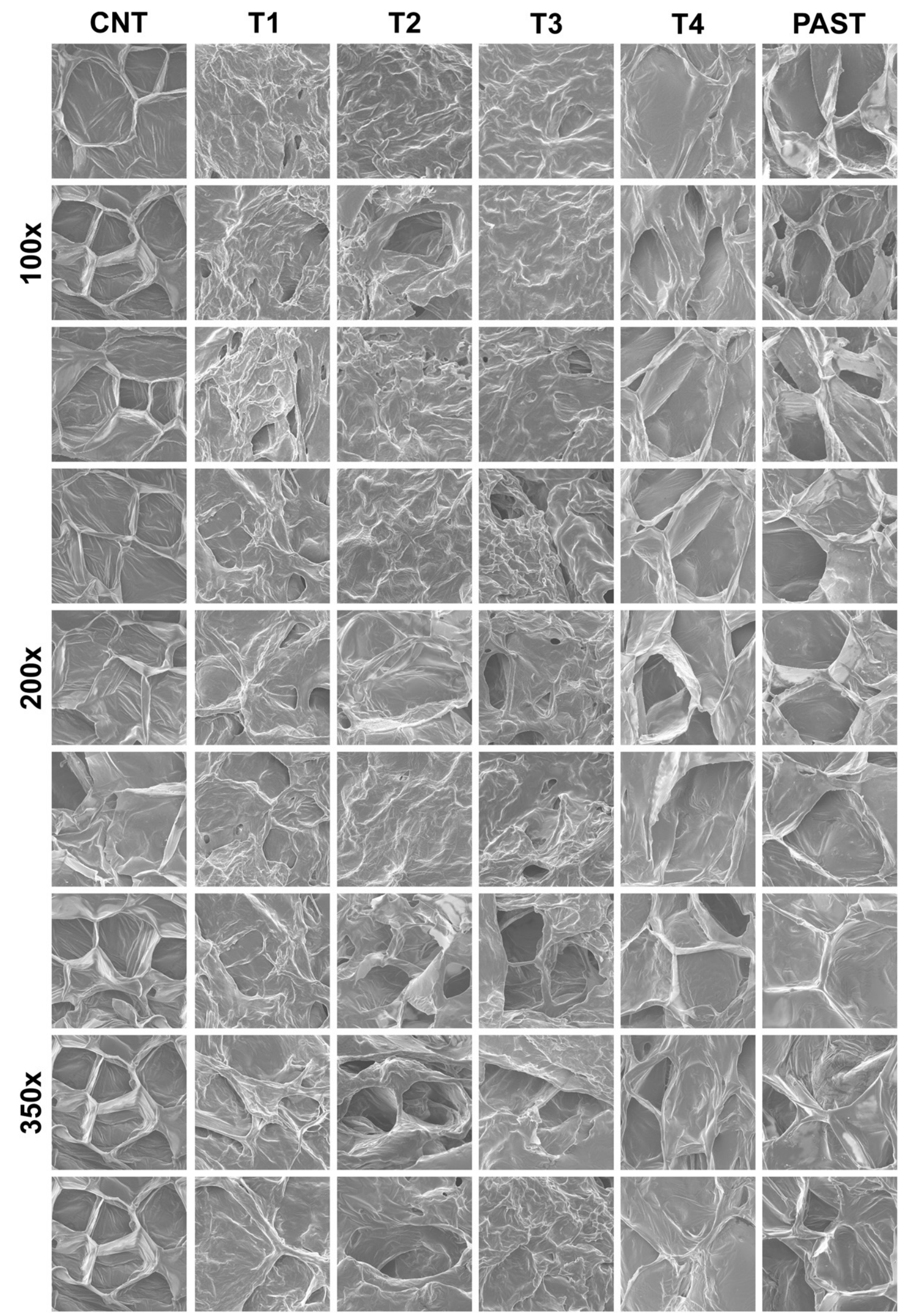

Fig. 4. Scanning electron microscopy crops evaluated at $100 \times, 200 \times$, and $350 \times$ of untreated, HHP-treated, and pasteurized red Lamuyo sweet pepper. 
A)

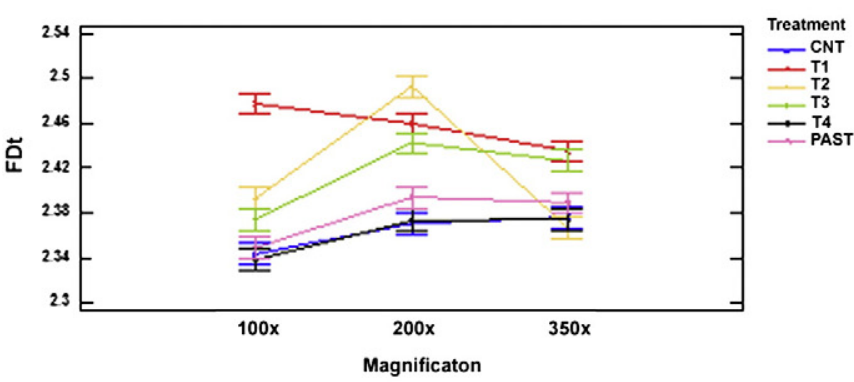

C)

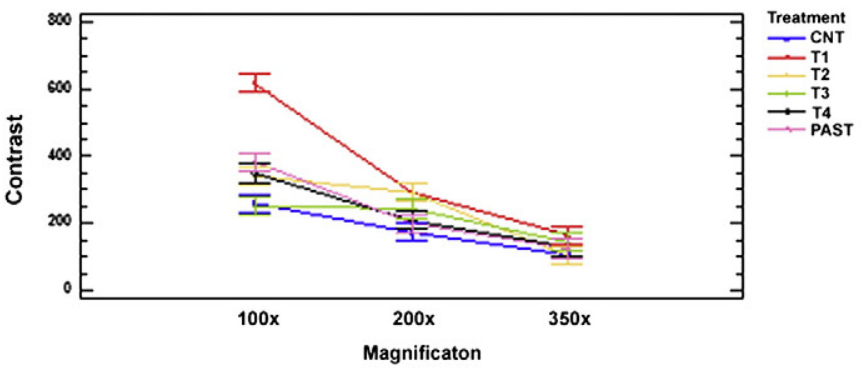

E)

Interactions and 95.0 percent LSD intervals
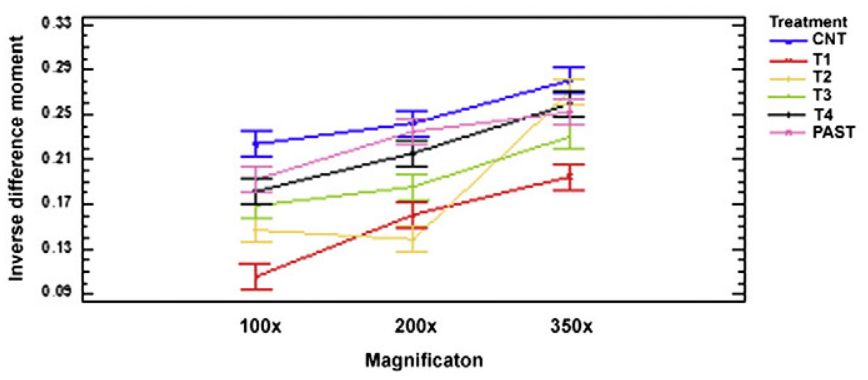

B)

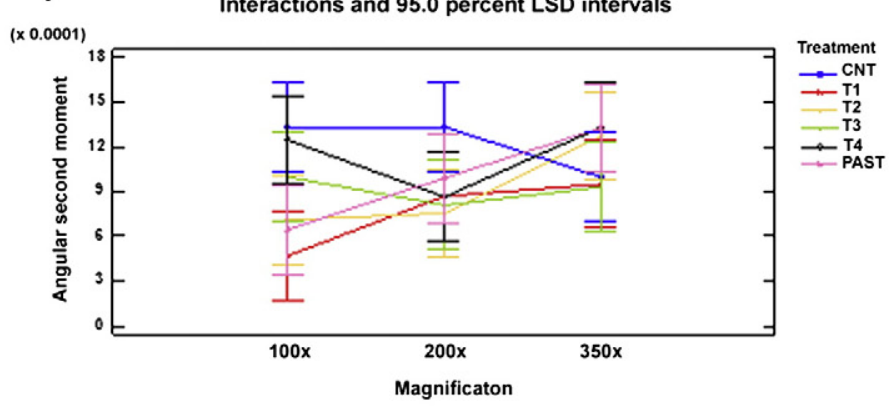

D)

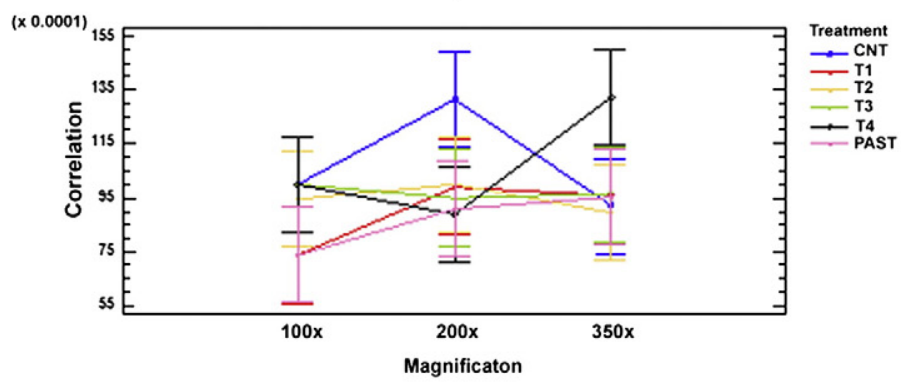

F)
Interactions and 95.0 percent LSD intervals

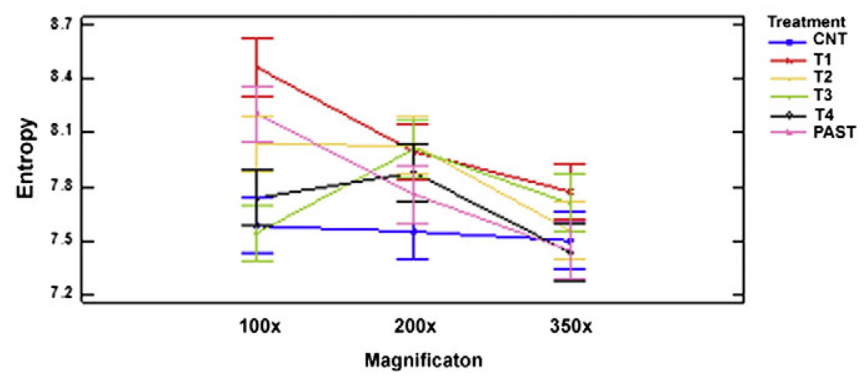

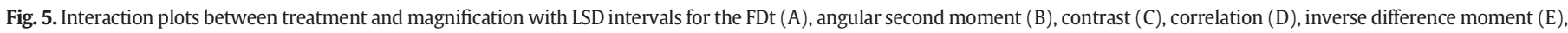
and entropy (F) of red Lamuyo sweet pepper.

biofilms (Yang et al., 2000), skin (Ou et al., 2014), and plastic greenhouses (Agüera et al., 2008). However, no many studies can be found that indicate the pertinence of the angular second moment on studying the texture of food products (Qiao, Ngadi, Wang, Gariépy, \& Prasher, 2007). Hence, angular second moment seems to be a textural parameter more appropriate to study the texture of no-food products surfaces.

Regarding the textural feature contrast, the statistical analysis of the results revealed a significant interaction $(p<0.05)$ between the applied treatment and the magnification used. Thus, it can be observed (Fig. 5C) that the micrographs of the CNT and T3 peppers had significantly lower contrast values $(p<0.05)$ at $100 \times$ magnification, while the peppers subjected to T1 treatment had significantly higher contrast values $(p<$ 0.05 ). The PAST, T2, and T4 treatments showed intermediate contrast values. When using the $200 \times$ magnification, treatments T1 and T2 resulted in significantly higher $(p<0.05)$ contrast values, while no significant differences $(p>0.05)$ were found for this parameter in the remaining the analyzed treatments. A high contrast value indicates a high degree of local variation (Mendoza, Dejmek, \& Aguilera, 2007; Park \& Chen, 2001), which is typical of rougher and more heterogeneous surfaces. This result would explain the higher contrast values observed for treatments T1 and T2 with rougher surfaces and the lower values observed for CNT peppers with a more homogenous surface. Finally, at $350 \times$ magnification, there was no statistically significant difference $(p>0.05)$ between the contrast values of the micrographs from peppers subjected to different treatments. This result again confirms that the magnification used to study the microstructure of red peppers has a clear influence on the textural parameters determined from micrographs, where cellular damage is clearer at smaller scales. In this case, we also observed that increased magnification resulted in a significant decrease $(p<0.05)$ in the contrast for all of the treatments considered in the study. Several publications can be found in the literature that indicate the pertinence of the textural feature contrast to numerically describe microstructural changes (Mendoza et al., 2007; Park \& Chen, 2001; Zheng, Sun, \& Zheng, 2007). By contrast, Laddi et al. (2013) when studying the contribution of different textural features (entropy, contrast, energy, homogeneity, and correlation) to estimate the tea quality concluded that contrast have no effect on tea quality. In the same way, Barrera et al. (2013) evaluated the mechanical damage on wheat starch granules and did not find significant differences of contrast parameter between damaged and native granules.

Similar to the angular second moment (Fig. 5D), when analyzing the correlation between micrographs from red peppers subjected to different preservation treatments, no statistically significant interaction $(p>$ 0.05 ) was found between the studied parameters, and no statistically significant difference $(p>0.05)$ was observed in the textural properties of any of the evaluated parameters (treatment and magnification). Just as 
for the angular second moment, its correlation did not appear to be an optimal parameter for evaluating the effect of HHP and PAST on the image texture of red peppers. Similar results obtained by Laddi et al. (2013) when studying the contribution of different textural features (entropy, contrast, energy, homogeneity, and correlation) to estimate the tea quality and concluded that correlation have no effect on tea quality. In the same way, Park and Chen (2001) obtained that correlation was not significant to differentiate unwholesome from wholesome poultry carcasses.

When analyzing the inverse difference moment (IDF) of the micrographs from red peppers subjected to different preservation treatments, the results showed a statistically significant interaction $(p<0.05)$ between the applied treatment and the magnification used. Thus, at $100 \times$ magnification (Fig. 5E), there was no statistically significant difference $(p>0.05)$ in the IDF for PAST, T3, and T4 treatment. However, the CNT pepper micrographs had significantly higher $(p<0.05)$ IDF values, and the treatment T1 resulted in significantly lower $(p<0.05)$ IDF values. These results are logical because it is known that IDF values indicate the degree of variation in image contrast, and high IDF values can be associated with homogeneous images (Barrera et al., 2013) such as the images obtained of the CNT red peppers. The elevated pressure tended to significantly increase $(p<0.05)$ the IDF, which was related to the lower structural damage observed with increased pressure. Similar results were obtained by Barrera et al. (2013) when they evaluated the mechanical damage on wheat starch granules. They concluded that the surface of damaged granules showed lower IDF values than native starch granules and suggested that the mechanical process decreased IDF. When using the 200× magnification, the micrographs from the CNT and PAST peppers had significantly higher IDF values $(p<0.05)$, while the peppers subjected to the T1 and T2 treatments had significantly lower IDF values $(p<0.05)$. Again, in this case, the increase in pressure tended to significantly increase the IDF values $(p<0.05)$ due to the decreased structural damage caused by the increased pressure. Finally, when the $350 \times$ magnification was used, treatment $\mathrm{T} 1$ yielded significantly lower IDF values $(p<0.05)$. This result was associated with the more heterogeneous surface obtained with treatment T1. It is also important to note that increasing the magnification used for making micrographs resulted in a significant increase $(p<0.05)$ of IDF for all of the analyzed treatments, which again shows that cellular damage is best observed at smaller scales. Hence, IDF seems to be a useful textural parameter to numerically describe microstructural changes. Numerous publications can be found in the literature that indicate this fact (Barrera et al., 2013; Laddi et al., 2013; Mendoza et al., 2007; Park \& Chen, 2001; Yang et al., 2000).

Finally, statistical analysis of the results revealed a statistically significant interaction $(p<0.05)$ between the applied treatment and the magnification used to analyze the entropy of the red pepper micrographs. Thus, it can be observed (Fig. 5F) that the micrographs corresponding to the CNT and T3 and T4 treatment peppers had significantly lower entropy $(p<0.05)$; there was no statistically significant difference ( $p>$ 0.05 ) between these groups. By contrast, the T1 and PAST treatments resulted in significantly higher $(p<0.05)$ entropy values. The higher entropy values obtained for $\mathrm{T} 1$ treatment red peppers could be related to the greater heterogeneity of its structure (Yang et al., 2000) since the more complex the images, the higher the entropy values (Barrera et al., 2013; Mendoza et al., 2007). At 200× magnification, there was no statistically significant difference $(p>0.05)$ in the entropy of the micrographs corresponding to treatments T1, T2, T3, T4, or PAST. Only the micrographs from the CNT peppers had significantly lower $(p<0.05)$ entropy values than the peppers treated by HHP; these micrographs were comparable to the PAST micrographs. The lower entropy values found in the images of the CNT and PAST peppers could be related to the greater homogeneity of their structures. (Mendoza et al., 2007; Yang et al., 2000). In the same way, Barrera et al. (2013) when evaluated the mechanical damage on wheat starch granules, obtained that damage granules showed higher entropy values than the native granules, suggesting that the mechanical process increased the entropy. When the $350 \times$ magnification was used, there was no statistically significant difference $(p>0.05)$ in the entropy of the micrographs corresponding to the different treatments analyzed, which could be expressed as cellular damage that is best observed at smaller scales. It should be noted here that the increase in magnification used in making micrographs did not result in a predictable trend in entropy, unlike in previous cases. That is, in some cases, the increase in magnification produced a significant decrease $(p<0.05)$ in entropy (T1, T2 and PAST), while in other cases, it did not cause significant changes $(p>0.05)$ in entropy (CNT, T3 and T4). Hence, entropy seems to be a useful textural parameter to numerically describe microstructural changes. Numerous publications can be found in the literature that indicate this fact (Barrera et al., 2013; Laddi et al., 2013; Mendoza et al., 2007; Park \& Chen, 2001; Yang et al., 2000).

\section{Conclusions}

All the preservation treatments studied, whether PA or HHP, caused structural changes in red Lamuyo-type sweet pepper tissues, but HHP T4 and PA were the treatments that had the least impact on the microstructure. Structural modifications in the red pepper tissues caused by HHP and PAST led to variations in the area, perimeter, circularity, and Feret diameter of the pepper cells, changes in the distribution of cell size and modification of the image texture parameters. Of the studied texture parameters, some were more relevant than others for characterizing the effects of HHP and PAST on the texture of the red peppers: FDt, contrast, IDF, and entropy. By contrast, the magnification at which the texture was evaluated was a parameter to consider given that the cellular damage is best observed at smaller scales. Anyway, it would be recommendable to investigate other magnifications in future research. There appears to be a relationship between the structural damage to red pepper tissues resulting from preservation treatments and the effects on the functionality of the plant material such that the lower the structural damage (T4 and PAST treatments), the lower the impact on the functionality of the pepper. These results suggest the relevance of image analysis as a quantitative and non-invasive technique that could be related in future studies to the biological functionality of products subject to HHP.

\section{Acknowledgments}

The authors wish to acknowledge the Spanish Ministry of Science and Innovation for the financial support (project AGL2011-30064C02) and to the Universitat Politècnica de València (UPV) for the FPI grant given to María Hernández Carrión. The authors also thank University of La Sabana for let Maria Hernández Carrión to do a stay of research and for access to their materials and methods. Moreover, the authors wish to thank Elsevier for assistance with the English manuscript.

\section{References}

Abdullah, M. Z., Aziz, S. A., \& Dos Mohamed, A. M. (2000). Quality inspection of bakery products using a color-based machine vision system. Journal of Food Quality, 23(1), 39-50.

Agüera, F., Aguilar, F. J., \& Aguilar, M. A. (2008). Using texture analysis to improve perpixel classification of very high resolution images for mapping plastic greenhouses. ISPRS Journal of Photogrammetry and Remote Sensing, 63(6), 635-646.

Aguilera, J. M. (2007). Microstructure and food product engineering. Food Technology, 54(11), 56-65.

Arzate-Vázquez, I., Chanona-Pérez, J. J., Calderón-Domínguez, G., Terres-Rojas, E., GaribayFebles, V., Martínez-Rivas, A., et al. (2012). Microstructural characterization of chitosan and alginate films by microscopy techniques and texture image analysis. Carbohydrate Polymers, 87(1), 289-299.

Barrera, G. N., Calderón-Domínguez, G., Chanona-Pérez, J., Gutiérrez-López, G. F., León, A. E., \& Ribotta, P. D. (2013). Evaluation of the mechanical damage on wheat starch granules by SEM, ESEM, AFM and texture image analysis. Carbohydrate Polymers, 98(2), 1449-1457.

Chen, W., Yuan, S., Hsiao, H., \& Hsieh, C. (2001). Algorithms to estimating fractal dimension of texture images. IEEE International Conference on Acoustics, Speech and Signal Processing, Vol. 3. (pp. 1541-1544) (Hong Kong (China)). 
Deepa, N., Kaur, C., George, B., Singh, B., \& Kapoor, H. C. (2007). Antioxidant constituents in some sweet pepper (Capsicum annuum L.) genotypes during maturity. LWT - Food Science and Technology, 40(1), 121-129.

Devlieghere, F., Vermeiren, L., \& Debevere, J. (2004). New preservation technologies: Possibilities and limitations. International Dairy Journal, 14(4), 273-285.

Duma, M., \& Alsina, I. (2012). The content of plant pigments in red and yellow bell peppers. Scientific Papers Series B Horticulture, 56, 105-108.

Ersahin, S., Gunal, H., Kutlu, T., Yetgin, B., \& Coban, S. (2006). Estimating specific surface area and cation exchange capacity in soils using fractal dimension of particle-size distribution. Geoderma, 136(3-4), 588-597.

Faustino, J. M. F., Barroca, M. J., \& Guiné, R. P. F. (2007). Study of the drying kinetics of green bell pepper and chemical characterization. Food and Bioproducts Processing 85(3), 163-170.

Gonzales-Barron, U., \& Butler, F. (2008). Fractal texture analysis of bread crumb digital images. European Food Research and Technology, 226(4), 721-729.

Gonzalez, R. C., Woods, R. E., \& Eddins, S. L. (2003). Digital image processing using MATLAB. New Jersey: Prentice Hall (Chapter 11).

Grillo, O., Rizzo, V., Saccone, R., Fallico, B., Mazzaglia, A., Venora, G., et al. (2014). Use of image analysis to evaluate the shelf life of bakery products. Food Research International, 62, 514-522.

Haralick, R. M., Shanmugam, K., \& Dinstein, I. (1973). Textural features for image classification. IEEE Transactions on Systems, Man, and Cybernetics, 3(6), 610-621.

Haralick, R. M., \& Shapiro, L. G. (1992). Computer and robot vision, Vol. 1, Addison-Wesley.

Hernández-Carrión, M., Hernando, I., \& Quiles, A. (2014). High hydrostatic pressure treatment as an alternative to pasteurization to maintain bioactive compound content and texture in red sweet pepper. Innovative Food Science and Emerging Technologies, 26, 76-85.

Hernández-Carrión, M., Vázquez-Gutiérrez, J. L., Hernando, I., \& Quiles, A. (2014). Impact of high hydrostatic pressure and pasteurization on the structure and the extractability of bioactive compounds of persimmon "Rojo Brillante". Journal of Food Science, 79(1), C32-C38.

Laddi, A., Sharma, S., Kumar, A., \& Kapur, P. (2013). Classification of tea grains based upon image texture feature analysis under different illumination conditions. Journal of Food Engineering, 115(2), 226-231.

Llorca, E., Puig, A., Hernando, I., Salvador, A., Fiszman, S. M., \& Lluch, M. A. (2001). Effect of fermentation time on texture and microstructure of pickled carrots. Journal of the Science of Food and Agriculture, 81(15), 1553-1560.

López-García, F., Andreu-García, G., Blasco, J., Aleixos, N., \& Valiente, J. -M. (2010). Automatic detection of skin defects in citrus fruits using a multivariate image analysis approach. Computers and Electronics in Agriculture, 71(2), 189-197.

McClements, J. M. J., Patterson, M. F., \& Linton, M. (2001). The effect of growth stage and growth temperature on high hydrostatic pressure inactivation of some psychrotrophic bacteria in milk. Journal of Food Protection, 64(4), 514-522.

Mendoza, F., Dejmek, P., \& Aguilera, J. M. (2007). Colour and image texture analysis in classification of commercial potato chips. Food Research International, 40(9), 1146-1154.

Norton, T., \& Sun, D. W. (2008). Recent advances in the use of high pressure as an effective processing technique in the food industry. Food and Bioprocess Technology, 1(1), 2-34.

Ou, X., Pan, W., \& Xiao, P. (2014). In vivo skin capacitive imaging analysis by using grey level co-occurrence matrix (GLCM). International Journal of Pharmaceutics, $460(1-2), 28-32$

Park, B., \& Chen, Y. R. (2001). AE-Automation and emerging technologies: Co-occurrence matrix texture features of multi-spectral images on poultry carcasses. Journal of Agricultural Engineering Research, 78(2), 127-139.

Pedreschi, F., Mery, D., Mendoza, F., \& Aguilera, J. M. (2004). Classification of potato chips using pattern recognition. Journal of Food Science, 69(6), E264-E270.

Penna, A. L. B., Subbarao, G., \& Barbosa-Cánovas, G. V. (2007). High hydrostatic pressure processing on microstructure of probiotic low-fat yogurt. Food Research International, 40(4), 510-519.

Qiao, J., Ngadi, M. O., Wang, N., Gariépy, C., \& Prasher, S. O. (2007). Pork quality and marbling level assessment using a hyperspectral imaging system. Journal of Food Engineering, 83(1), 10-16.

Quevedo, R., Jaramillo, M., Díaz, O., Pedreschi, F., \& Aguilera, J. M. (2009). Quantification of enzymatic browning in apple slices applying the fractal texture Fourier image. Journal of Food Engineering, 95(2), 285-290.
Quevedo, R., López, G. C., Aguilera, J. M., \& Cadoche, L. (2002). Description of food surfaces and microstructural changes using fractal image texture analysis. Journal of Food Engineering, 53(4), 361-371.

Quintanilla-Carvajal, M. X., Meraz-Torres, L. S., Alamilla-Beltrán, L., Chanona-Pérez, J. J., Terres-Rojas, E., Hernández-Sánchez, H., et al. (2011). Morphometric characterization of spray-dried microcapsules before and after a-tocopherol extraction. Revista Mexicana de Ingenieria Quimica, 10, 301-312.

Rastogi, N. K., Raghavarao, K. S. M. S., Balasubramaniam, V. M., Niranjan, K., \& Knorr, D. (2007). Opportunities and challenges in high pressure processing of foods. Critical Reviews in Food Science and Nutrition, 47(1), 69-112.

Rocha, A., Hauagge, D. C., Wainer, J., \& Goldenstein, S. (2010). Automatic fruit and vegetable classification from images. Computers and Electronics in Agriculture, 70(1), 96-104.

Romano, G., Argyropoulos, D., Nagle, M., Khan, M. T., \& Müller, J. (2012). Combination of digital images and laser light to predict moisture content and color of bell pepper simultaneously during drying. Journal of Food Engineering, 109(3), 438-448.

Russ, J. C. (2007). The image processing handbook (5th ed.). Boca Ratón: CRC Press.

Serrano, M., Zapata, P. J., Castillo, S., Guillén, F., Martínez-Romero, D., \& Valero, D. (2010) Antioxidant and nutritive constituents during sweet pepper development and ripening are enhanced by nitrophenolate treatments. Food Chemistry, 118(3), 497-503.

Sonka, M., Hlavac, V., \& Boyle, R. (2008). Image processing, analysis, and machine vision (3rd ed.). Toronto: Thomson Learning.

Sun, D. W. (2008). Computer vision technology for food quality evaluation (1st ed.). San Diego: Academic Press/Elsevier.

Tang, D., \& Marangoni, A. (2006). Computer simulation of fractal dimensions of fat crystal networks. Journal of the American Oil Chemists' Society, 83(4), 309-314.

Tedjo, W., Taiwo, K. A., Eshtiaghi, M. N., \& Knorr, D. (2002). Comparison of pretreatment methods on water and solid diffusion kinetics of osmotically dehydrated mangos. Journal of Food Engineering, 53(2), 133-142.

Trejo Araya, X. I., Hendrickx, M., Verlinden, B. E., Van Buggenhout, S., Smale, N. J., Stewart C., et al. (2007). Understanding texture changes of high pressure processed fresh carrots: A microstructural and biochemical approach. Journal of Food Engineering, 80(3) 873-884.

Valous, N. A., Mendoza, F., Sun, D. -W., \& Allen, P. (2009). Texture appearance characterization of pre-sliced pork ham images using fractal metrics: Fourier analysis dimension and lacunarity. Food Research International, 42(3), 353-362.

Vázquez-Gutiérrez, J. L., Hernández-Carrión, M., Quiles, A., Hernando, I., \& Pérez-Munuera, I. (2012). Impact of high hydrostatic pressures on the structure, diffusion of soluble compounds and textural properties of persimmon 'Rojo Brillante'. Food Research International, 47(2), 218-222.

Vázquez-Gutiérrez, J. L., Plaza, L., Hernando, I., Sánchez-Moreno, C., Quiles, A., De Ancos, B et al. (2013). Changes in the structure and antioxidant properties of onions by high pressure treatment. Food \&' Function, 4, 586-591.

Vega-Gálvez, A., Uribe, E., Perez, M., Tabilo-Munizaga, G., Vergara, J., Garcia-Segovia, P. et al. (2011). Effect of high hydrostatic pressure pretreatment on drying kinetics, antioxidant activity, firmness and microstructure of aloe vera (Aloe barbadensis Miller) gel. LWT - Food Science and Technology, 44(2), 384-391.

Venora, G., Grillo, O., Ravalli, C., \& Cremonini, R. (2009). Identification of Italian landraces of bean (Phaseolus vulgaris L.) using an image analysis system. Scientia Horticulturae, 121(4), 410-418.

Venora, G., Grillo, O., \& Saccone, R. (2009). Quality assessment of durum wheat storage centres in Sicily: Evaluation of vitreous, starchy and shrunken kernels using an image analysis system. Journal of Cereal Science, 49(3), 429-440.

Venora, G., Grillo, O., Shahin, M. A., \& Symons, S. J. (2007). Identification of Sicilian landraces and Canadian cultivars of lentil using an image analysis system. Food Research International, 40(1), 161-166.

Yang, X., Beyenal, H., Harkin, G., \& Lewandowski, Z. (2000). Quantifying biofilm structure using image analysis. Journal of Microbiological Methods, 39(2), 109-119.

Zheng, C., Sun, D. -W., \& Zheng, L. (2007). A new region-primitive method for classification of colour meat image texture based on size, orientation, and contrast. Meat Science, 76(4), 620-627.

Zhuang, Y., Chen, L., Sun, L., \& Cao, J. (2012). Bioactive characteristics and antioxidant activities of nine peppers. Journal of Functional Foods, 4(1), 331-338. 